\title{
Incidence and diversity of the fungal genera Aspergillus and Penicillium in Portuguese almonds and chestnuts
}

\author{
Paula Rodrigues • Armando Venâncio • Nelson Lima
}

Accepted: 22 May 2013 /Published online: 14 June 2013

(C) KNPV 2013

\begin{abstract}
Almonds (Prunus dulcis (Miller) D.A. Webb) and European (sweet) chestnuts (Castanea sativa Miller) are of great economic and social impact in Mediterranean countries, and in some areas they constitute the main income of rural populations. Despite all efforts to control fungal contamination, toxigenic fungi are ubiquitous in nature and occur regularly in worldwide food supplies, and these nuts are no exception. This work aimed to provide knowledge on the general mycobiota of Portuguese almonds and chestnuts, and its evolution from field to the end of storage. For this matter, 45 field chestnut samples and 36 almond samples (30 field samples and six storage samples) were collected in Trás-os-Montes, Portugal. All fungi belonging to genus Aspergillus were isolated and identified to the section level. Fungi representative of other genera were identified to the genus level. In the field, chestnuts were mainly contaminated with the genera Fusarium,
\end{abstract}

P. Rodrigues $(\bowtie)$

CIMO/School of Agriculture of the Polytechnic Institute of Bragança, Campus de Santa Apolónia,

5301-855 Bragança, Portugal

e-mail: prodrigues@ipb.pt

A. Venâncio $\cdot$ N. Lima

IBB-Institute for Biotechnology and Bioengineering, Centre for Biological Engineering, Universidade do Minho, Campus de Gualtar, 4710-057 Braga, Portugal
A. Venâncio
e-mail: avenan@deb.uminho.pt
N. Lima
e-mail: nelson@ie.uminho.pt

Cladosporium, Alternaria and Penicillium, and the genus Aspergillus was only rarely found, whereas almonds were more contaminated with Aspergillus. In almonds, Aspergillus incidence increased significantly from field to the end of storage, but diversity decreased, with potentially toxigenic isolates belonging to sections Flavi and Nigri becoming more significant and widespread throughout storage. These fungi were determined to be moderately associated, which can be indicative of mycotoxin co-contamination problems if adequate storage conditions are not secured.

Keywords Aspergillus $\cdot$ Penicillium $\cdot$ Field fungi Storage fungi $\cdot$ Fungal association

\section{Introduction}

Almonds (Prunus dulcis (Miller) D.A. Webb, synonym Amygdalus communis L.) and European (sweet) chestnuts (Castanea sativa Miller) are of great economic and social impact in Mediterranean countries, and in Portugal they constitute the main income of rural populations. Portugal has a typical Mediterranean climate, generally characterised by long periods of high temperatures and moderate-to-scarce rainfall. Almonds are extremely dry nuts produced under highly stressful environmental conditions, in regions where the maturation and harvest period corresponds to a hot and dry summer. On the other hand, chestnuts are produced under more cold and humid conditions. Under such conditions, tree nuts, and mostly almonds, are known to be targets of 
infection for a variety of fungi that can induce spoilage or produce mycotoxins.

Despite all efforts to control fungal contamination, toxigenic fungi are ubiquitous in nature and occur regularly in worldwide food supplies. Fungal growth and mycotoxin production only occur under favourable conditions, which vary for each species depending on adaptability. Food intrinsic parameters associated to environmental factors are responsible for the spectrum of contaminating and dominating mycobiota.

Genera like Botrytis, Cladosporium and Rhizopus, are major spoilage fungi in a variety of nuts, but they are not known to produce significant mycotoxins. On the other hand, Aspergillus, Fusarium and Penicillium include species capable of producing a wide range of mycotoxins. These fungi have generally been reported as important contaminants in almonds and chestnuts (see Rodrigues et al. 2012b for review).

Alternaria, Cladosporium and Fusarium are traditionally classified as field fungi. These fungi usually require temperatures around 20 to $25^{\circ} \mathrm{C}$ and $\mathrm{a}_{\mathrm{W}}>0.85$ for active growth, but they grow optimally at $\mathrm{a}_{\mathrm{W}}$ near 0.99. Aspergillus and Penicillium are major representatives of the so-called storage fungi. The minimal necessary $\mathrm{a}_{\mathrm{W}}$ for most Aspergillus and Penicillium species is $0.75-0.85$, but they generally grow optimally at $\mathrm{a}_{\mathrm{W}} 0.93-0.98$. Aspergillus can grow at $\mathrm{a}_{\mathrm{W}}$ as low as 0.73 and is generally more adapted to temperatures of $30-40{ }^{\circ} \mathrm{C}$, whereas Penicillium needs at least $0.78-$ $0.80 \mathrm{a}_{\mathrm{W}}$ and grows optimally at $25-30{ }^{\circ} \mathrm{C}$ (Rosso and Robinson 2001; Filtenborg et al. 2004; Magan 2006).

Knowledge of the mycological contamination of a given matrix helps understanding the fungal dynamics throughout production and storage, and gives important information for the determination of effective control measures for reducing the probability of preand post-harvest mycotoxin contamination. Thus, the intention of this work was to determine the mycobiota associated with Portuguese almonds and chestnuts from the field to the end of storage.

\section{Materials and methods}

Study area-geographic and climatic characterisation

Sampling of chestnuts and almonds for mycobiota surveys were conducted in the main producing regions of Portugal.
Chestnut sampling was conducted in Santa Comba de Rossas, District of Bragança, in the northeastern Portuguese sub-region of Alto Trás-os-Montes (Fig. 1). This area is responsible for $85 \%$ of the national area devoted to chestnut production, and corresponds to more than $80 \%$ of the national production. It integrates three chestnut Protected Denomination of Origin (Denominação de Origem Protegida, D.O.P)—Terra Fria, Padrela and Soutos da Lapa. It is located at a latitude of $41^{\circ} 49^{\prime} \mathrm{N}$, longitude of $06^{\circ} 45^{\prime} \mathrm{W}$ and an altitude of approximately $720 \mathrm{~m}$. Bragança is characterised by a humid climate with a yearly rainfall of $741.1 \mathrm{~mm}$, with moderate water deficit during summer, and great excess in winter. The summer is warm, with mean temperatures around $21^{\circ} \mathrm{C}$, and the winter is cold, with temperatures frequently dropping below $0{ }^{\circ} \mathrm{C}$.

Almond sampling was conducted in Moncorvo, in the south of Bragança District, sub-region Douro, at a latitude of $41^{\circ} 04 \mathrm{~N}$, longitude of $07^{\circ} 01^{\prime} \mathrm{W}$ and an altitude of approximately $410 \mathrm{~m}$ (Fig. 1). This region represents more than $60 \%$ of the national production of almonds and integrates one almond D.O.P., Douro. Moncorvo is characterised by hot summers, with mean temperatures around $24{ }^{\circ} \mathrm{C}$, but $40{ }^{\circ} \mathrm{C}$ being registered with some frequency during July and August. Moncorvo registers mean temperatures of $6{ }^{\circ} \mathrm{C}$ in the cold months of December and January, and a yearly rainfall of $520.1 \mathrm{~mm}$, with extreme water deficit during summer.

\section{Sampling plans}

\section{Almond field samples}

Three almond orchards, approximately $500 \mathrm{~m}$ apart from each other, were selected for field sampling. Five actively producing trees per orchard were selected as sampling points, in a total of 15 sampling spots.

Two samples were taken from each sampling spot, regarding two consecutive crops: 2007 (Tree/07) and 2008 (Tree/08). The sampling time-points (06/09/2007 and 12/09/2008) corresponded to the day before the beginning of harvest. Samples were composed of 50 nuts, randomly collected from the tree canopy. Nuts were collected by hand and put in a C4 craft paper envelope $(229 \times 324 \mathrm{~mm})$. The envelope was immediately sealed and stored in a portable refrigerator. Hands were disinfected with $70 \%$ ethanol between each sampling spot. Samples arrived at the laboratory no more than $3 \mathrm{~h}$ later. 


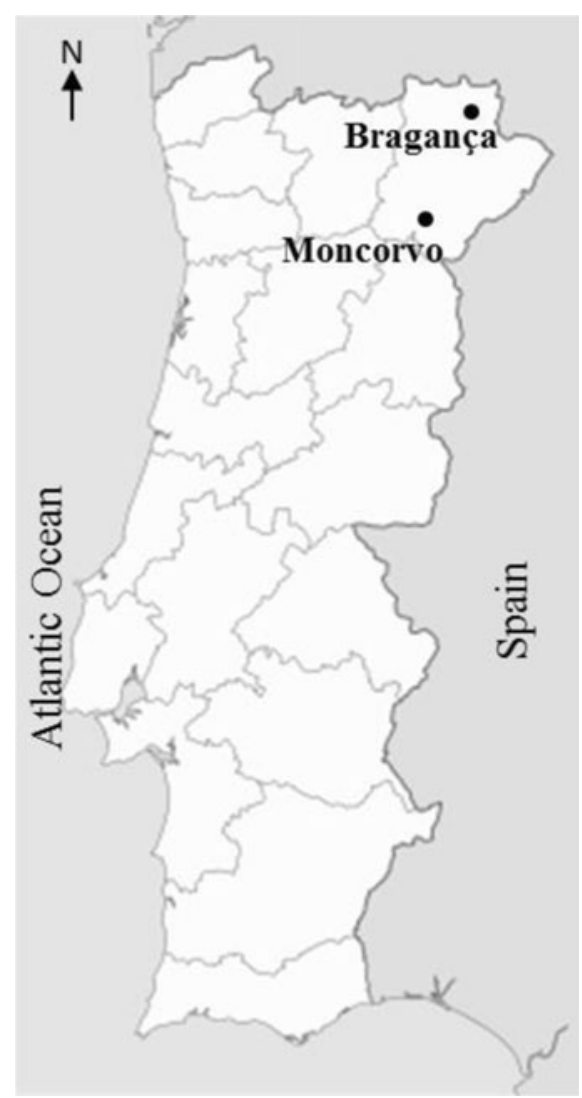

Fig. 1 Location of the sampling regions in Portugal mainland

\section{Almond storage samples}

Sampling during storage took place for the 2008 crop only. From 13/09/2008 onwards, almonds were continuously collected by the producer, spread in the warehouse and left to dry. On 24/10/2008, almonds began to be stockpiled in $50 \mathrm{~kg}$ bags (by order of arrival). Two bags from different parts of the pile were sampled. Approximately $2 \mathrm{~kg}$ of inshell almonds were collected from various parts of each selected bag. Samples were collected every 3 months, until the almonds were expedited. So, after the first sampling time-point (24/10/2008), two other samples were taken from the same bags (16/01/2009 and 20/03/2009).

\section{Chestnut field samples}

For chestnuts, a similar sampling procedure was followed, but with different time-points for sample collections. Chestnut samples were only collected in 2007, and three samples were taken from each sampling spot: the first sampling time-point coincided with the beginning of cropping (01/11/2007), when the farmer began to crop fallen nuts from the ground. At this time-point, one sample of nuts was collected from the ground (Gr1/07) and one sample from the tree (Tree/07). The second sampling time-point occurred at 20/11/2007, and corresponded to the period when all nuts had fallen from the trees and the farmer began to crop them mechanically (Gr2/07). From the first to the second sampling time-point, the farmer collected the fallen chestnuts erratically, so sampled chestnuts from 20/11/2007 had been on the ground between one to 20 days. At this time-point, only one sample of chestnuts was taken from the ground. Chestnuts were not sampled during storage since, in 2007, fruits were only stored for a few days and immediately dispatched.

Mycological analysis

From each sample, fruits were taken randomly from the envelopes using a sterile forceps. For field samples, six fruits per sample were plated, three with the shell (for determination of superficial contamination) and three without the shell (for determination of internal contamination), in a total of 45 in-shell fruits and 45 shelled fruits for each sampling time-point. For storage samples, 10 in-shell fruits and 10 shelled fruits per sample were plated. In-shell and shelled fruits were plated directly on Malt Salt Agar with $10 \% \mathrm{NaCl}$ (MSA10: Malt $20 \mathrm{~g} / \mathrm{l}$, Glucose $20 \mathrm{~g} / 1$, Peptone 1 g/l, NaCl 100 g/l, Agar 20 g/l) without surface disinfection, and covered with a thin layer of the same medium. Petri dishes were incubated in the dark, at $25-28{ }^{\circ} \mathrm{C}$, for 7 days. All plates were inspected after 3, 5 and 7 days of incubation, using a stereomicroscope (Nikon SMZ-U), to accompany fungal growth. After 7 days of incubation, all fungi belonging to genera Aspergillus and Penicillium were transferred to 9-cm Petri dishes containing $15 \mathrm{ml}$ of Malt Extract Agar (MEA: Malt $20 \mathrm{~g} / \mathrm{l}$, Glucose $20 \mathrm{~g} / \mathrm{l}$, Peptone $1 \mathrm{~g} / \mathrm{l}$, Agar $20 \mathrm{~g} / \mathrm{l}$ ) with an inoculation needle previously wetted in a sterile solution of $0.1 \%$ Tween 80. Isolates were grown in the dark for 7 days at $25^{\circ} \mathrm{C}$ and genus identification was confirmed based on macro- and micromorphological analysis (colony colour and characteristic conidiophore, respectively). Fungi belonging to the genus Aspergillus were further identified to the section level as described below.

Before identification, isolates were grown on MEA in the dark for 7 days at $25{ }^{\circ} \mathrm{C}$. From this culture, a loop full of spores was suspended in $500 \mu \mathrm{l}$ of $0.2 \%$ 
agar with $0.05 \%$ Tween 80 , and this suspension was used for three-point inoculations on 9-cm diameter Petri dishes containing MEA, CYA and CY20S as described in Rodrigues et al. (2012a). Cultures were incubated in the dark at $25{ }^{\circ} \mathrm{C}$. Whenever necessary, isolates were also cultured on Czapek-Dox Agar (CZ: the same as CYA, but without the Yeast Extract) at $25^{\circ} \mathrm{C}$ and CYA at $37{ }^{\circ} \mathrm{C}$ (CYA37).

Cultures from all media were analysed after 7 days of incubation for the following characters: colony growth, obverse and reverse colony colour, sclerotia and cleistothecia production and morphology, diffusible pigments and exudate production. Cultures on CYA were kept for 2 more weeks to confirm colony colour and, when teleomorphs were identified, to obtain mature ascospores. Colony colour and surface texture were compared with those presented in the taxonomic guides (Klich 2002; Samson et al. 2004).

Microscopic examination of spores and reproductive structures was made on actively sporulating material from cultures grown on CYA and MEA. Slides were prepared as wet mounts on distilled water (for pigmented fungi) or cotton blue (for hyaline fungi). Strongly sporulated material was previously washed with a drop of $96 \%$ ethanol and gently teased with the needle, to remove excessive conidia. Slides were examined with a compound light microscope (Leitz Labourlux 12) under bright field and Nomarski contrast. Stipes, vesicles, conidia and, when present, ascospores and cleistothecia were measured using the Measuring command of a previously calibrated Nikon Control Unit DS-LI/DS Camera. Stipe, conidia and ascospore surface textures as well as head seriation were observed with $1,000 \times$ magnitude amplification.

Identification followed the taxonomic keys and guides available for genus Aspergillus (Klich 2002; Samson et al. 2004). Some isolates were identified to the species level, while others were identified only to the section or aggregate levels. The taxonomic scheme proposed by Gams et al. (1985) was followed, since it is the one generally followed in the reference guides.

\section{Data analysis}

Samples were compared for overall distributions of fungi. Contingency tables were produced with fungal frequencies of infection, which were then compared by a two-tailed Fisher's exact test, with mean differences significant for $P<0.05$.
For the comparison of means of quantitative variables, samples were first tested for normal distribution by Shapiro-Wilk test (for $n<30$ ) or KolmogorovSmirnov test (for $n \geq 30$ ), and for homogeneity of variances by Levene's test. Whenever samples followed these criteria, variances were analysed by one-way ANOVA, and Multiple Comparisons between samples pairs were made by Bonferroni's test (for $n<30$ ) or Tukey's test (for $n \geq 30$ ). For samples failing the premise of homogeneity of variances, Tamhane's T2 test was applied. Whenever samples failed the two premises, normality and homogeneity of variances, samples were analysed pairwise by the non-parametric Mann-Whitney test (Maroco 2003). In all cases, the mean differences were significant at $P<0.05$.

The indices Richness and Simpson Diversity Index (SDI), as described by Zak and Willig (2004), were used to determine fungal diversity. Richness refers to the number of Aspergillus sections present in each sample. SDI was calculated as the reciprocal form of Simpson's Index $(1 / D)$, as follows:

$\mathrm{SDI}=1 / \sum\left(\mathrm{P}_{\mathrm{i}} / \mathrm{P}_{\mathrm{n}}\right)^{2}$

where $\sum(P i / P n)$ corresponds to D (Simpson's Index), $P_{i}$ is number of individuals of a given section, and $P_{n}$ is the total number of individuals.

Associations among fungi were tested pairwise by comparing observed values (the number of nuts infected by both fungi) with expected values (the product of the frequencies of each fungus alone), and by using Phi coefficient as measure of nominal association. Associations between fungal contamination and nominal non-dichotomous variables (origin, type of collection, stage of processing; $2 \times n$ table) were tested by using Cramer's $V$ as measure of nominal association (Maroco 2003). In the crosstabulations where at least one of the fungi contaminated $100 \%$ of the nuts, measures of association were not computed. On the other hand, associations between fungi with low or null incidence, namely those involving chestnut samples and sections Circumdati, Flavipedes, Fumigati and Nidulantes of almond samples, all resulted in extremely low Phi values and generally high P-values, meaning that no association could be established between those fungi. For that reason, those results are not shown. Also, association between a pair of intertwined variables is not valid, i.e., association cannot be established if one of the variables (e.g. Penicillium spp. or Aspergillus spp. 
contamination) is a component of the other variable (total contamination). Consequently, associations between the referred taxa were not computed.

Association values were interpreted as follows: very low association $0.00 \leq P h i$ or Cramer's $V<0.30$; low $0.30 \leq$ Phi or Cramer's $V<0.50$; moderate $0.50 \leq P h i$ or Cramer's $V<0.70$; high $0.70 \leq P h i$ or Cramer's $V<0.90$; and very high $0.90 \leq P h i$ or Cramer's $V \leq 1.00$ (adapted from http://www.westgard.com/lesson42.htm, accessed 20.09.2010). SPSS Statistics version 17.0 was used for all statistical analyses.

\section{Results}

Fungal incidence and diversity

In the present study, fungal incidence of chestnuts (field samples) and almonds (field and storage samples) was assessed. Contaminating fungi other than those belonging to the genera Aspergillus and Penicillium were analysed only qualitatively. The frequency of contamination with Penicillium species was determined only to the genus level. The isolates belonging to genus Aspergillus were identified to the section level, for both frequency of infection and number of individuals. Diversity was also assessed for the Aspergillus sections, through the Richness index, which in this case corresponds to the number of sections identified, and the Simpson Diversity Index (SDI), which reflects how many of those sections have significant impact on the diversity level of the samples. Table 1 lists the fungal incidence observed in chestnuts (field samples) and almonds (field and storage samples), for both in-shell (superficial contamination) and shelled (internal contamination) nuts. The number of Aspergillus isolated from these samples and the diversity indices Richness and Simpson Diversity Index (SDI) (relative to sections) are given in Table 2.

More than $80 \%$ of the nuts analysed in this study were contaminated with some fungi. The most common fungi were identified as belonging to the genera Alternaria, Aspergillus, Botrytis, Cladosporium, Fusarium and Penicillium. Aspergillus isolates were identified in nine different sections: Aspergillus (represented by the highly xerophilic species belonging to the teleomorphic genus Eurotium) was the most disseminated section, with the highest number of isolates (210 isolates), followed by
Versicolores (102 isolates), Wentii (76 isolates), Nigri (56 isolates) and Flavi (37 isolates). Four of the nine sections were only rarely isolated: Circumdati (10 isolates), Flavipedes (seven isolates), Nidulantes (two isolates) and Fumigati (one isolate).

In chestnut samples, $100 \%$ of superficial contamination was observed. Alternaria, Botrytis, Cladosporium, Fusarium and unidentified yeasts were the most commonly found fungi (data not quantified). Penicillium and Aspergillus were present at lower levels, the first being generally more frequent than the latter (Table 1). In fact, genus Aspergillus was very weakly represented in chestnuts, in terms of frequency of contamination as well as in number and variety of propagules. Chestnuts contamination with Aspergillus included only three sections: Nigri (60\% of the isolates), Aspergillus (26.6\%) and Wentii (13.3\%). Chestnuts showed a mean number of Aspergillus per nut (taken as the average of the three samples) of 0.07 and 0.04 for in-shell and shell nuts, respectively. Richness and SDI, which varied from 0 to 2 , reflect the extremely low diversity of these samples relative to this genus.

Chestnuts collected from the ground were shown to be highly contaminated by unidentified yeasts and by filamentous fungi mainly belonging to the genera Alternaria, Botrytis, Cladosporium and Fusarium. Penicillium represented an incidence of $45 \%$ in inshelled nuts, whereas aspergilli were identified in only $7 \%$ of the analysed nuts. From the Aspergillus genus, sections Nigri, Aspergillus and Wentii were the only ones present.

Chestnut samples were pairwise compared in order to determine the influence of the time and method of chestnut collection on fungal contamination. In general, chestnuts that had been on the ground for up to 3 weeks (sample Gr2/07) showed higher levels of contamination with overall fungi and Penicillium than the other samples, but were less contaminated with Aspergillus spp. In-shell nuts were $100 \%$ contaminated, regardless of the sample. Contamination with Penicillium spp. was higher, but not significantly, in sample Gr2/07 than in samples Tree1/07 and Gr1/07 ( $P=0.205$ and $P=0.139$, respectively). As opposed to that, Aspergillus spp. were less frequent in sample Gr2/07 (0 \% incidence). In this particular case, Penicillium and Aspergillus incidences seem to have an opposite trend. Considering Aspergillus sections, contamination was very low, and no significant differences were registered between samples. 
20

Eur J Plant Pathol (2013) 137:197-209

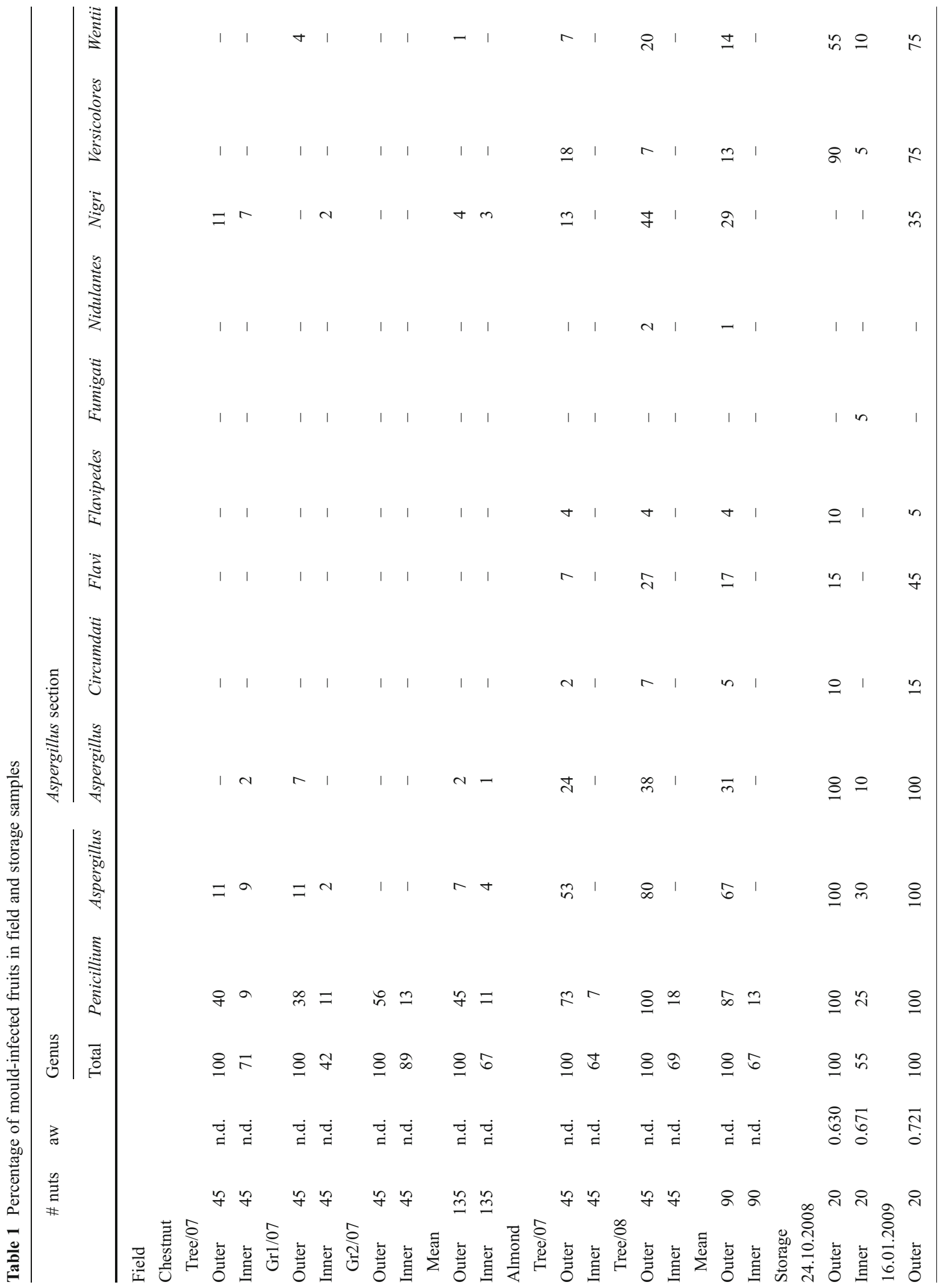

型 Springer 
Sample Gr1/07 showed significantly less overall internal contamination (shelled nuts) than samples Tree1/07 and $G r 2 / 07(P<0.001)$, but no other significant differences were registered $(P>0.117)$. When comparing inshell with shelled nuts, shelled nuts had significantly less contamination with general fungi and Penicillium than inshell nuts, for all samples $(P<0.026)$. On the other hand, contamination with Aspergillus was not significantly different between in-shell and shelled nuts $(P>0.361)$.

Field-collected almonds showed strong contamination with the same fungi identified in chestnuts, mainly Cladosporium, but, besides those, Aspergillus species were also found to be important superficial contaminants of almonds (Table 1). Eight different Aspergillus sections were identified, with the most common being sections Aspergillus, Nigri and Flavi (Table 2). Nevertheless, no internal contamination was associated with aspergilli.

When considering storage-collected almonds, Penicillium and Aspergillus were the predominant contaminants, both externally (in-shell) and internally (shelled). In fact, other genera like Cladosporium and Fusarium were still present, but were considerably less frequent than in field-collected nuts. Even though total contamination was not significantly different from field samples (100\% in most cases), Aspergillus became significantly more important as a contaminant. This was evidenced by the average number of Aspergillus propagules per nut in almonds harvested in 2008. External contamination with Aspergillus increased from 1.7 in the field to 5.6 in storage, and internal contamination increased from 0 to 0.4 . On the other hand, the Richness suffered a reduction from 8 to 5.5 (with only three sections being representative) on in-shell nuts, but increased from 0 to 2 on shelled nuts.

Contamination with Aspergillus spp. significantly increased throughout the dry period of storage, due mainly to xerophilic sections Aspergillus, Versicolores and Wentii, whereas sections Flavi and Nigri, slightly less xerophilic, were only present during the storage stage where $\mathrm{RH}$ was higher. This was also the stage with the highest Richness index, with seven sections identified, even if only four were of significance to the overall population.

Associations among fungi

Tables 3 and 4 list the association between fungi (Phi coefficient), and the corresponding significance (P-value), for in-shell and shelled nuts, respectively. 


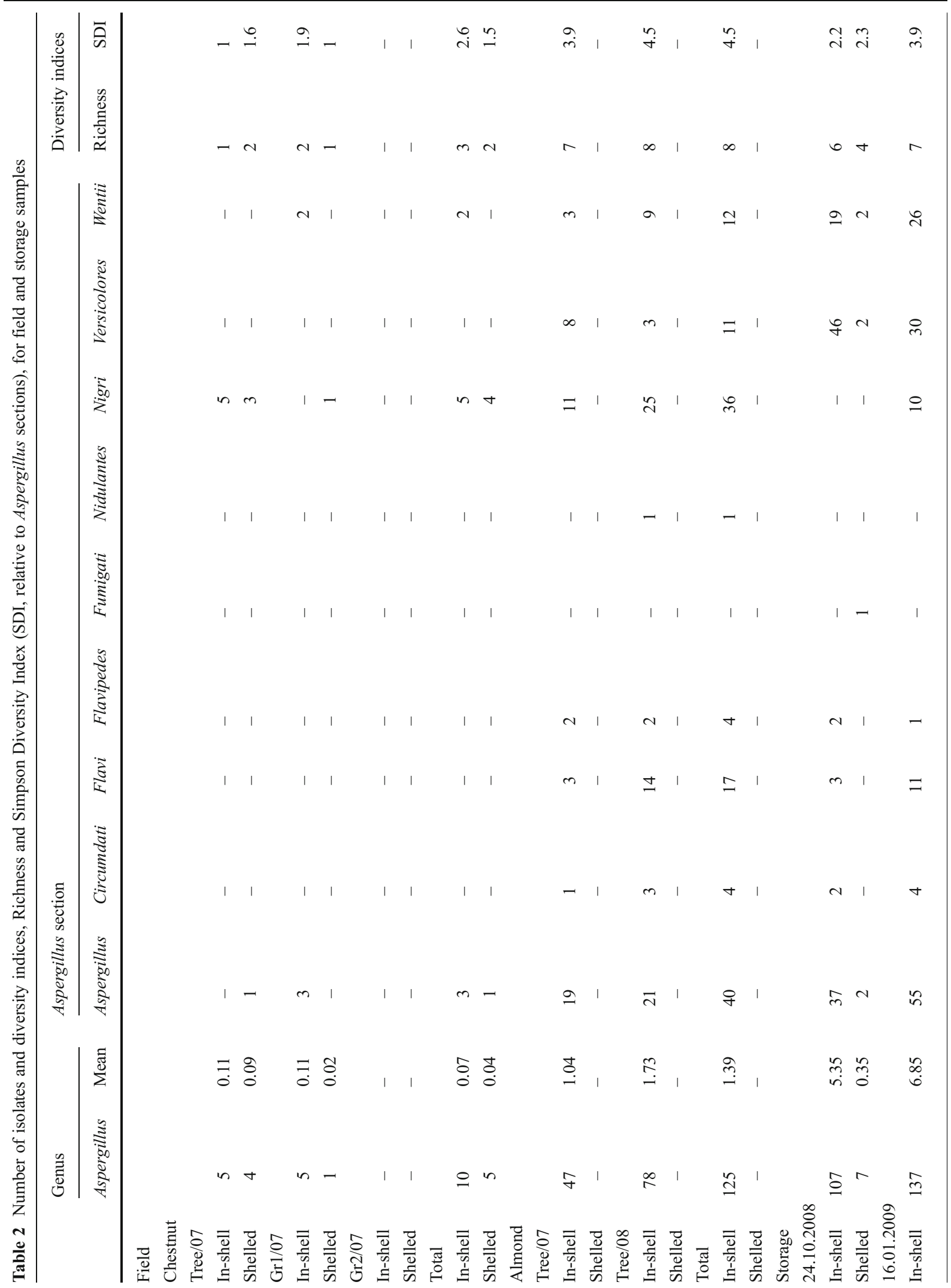


Associations among fungi were almost always positive and generally highly significant, but high levels of association between fungi were rare. In terms of superficial contamination, section Flavi correlated mostly (but only moderately) with section Nigri (Table 3), meaning that nuts superficially contaminated with representatives of section Flavi are more likely to be co-contaminated with section Nigri than with any other fungus. On the other hand, for internal contamination Penicillium spp. were highly and significantly correlated with Aspergillus spp. and section Aspergillus, but sections Flavi and Nigri associated very poorly with each other (Table 4).

\section{Discussion}

In a general overview, more than $80 \%$ of the nuts analysed in this study were contaminated with fungi. The most common fungi were identified as belonging to the genera Alternaria, Aspergillus, Botrytis, Cladosporium, Fusarium and Penicillium. Filtenborg et al. (2004) refer that normally less than 10 species are present in a given food commodity, and only 1-3 dominate and are responsible for spoilage. Even though mycobiota varied in terms of nuts and stage of production, we observed that, from among all fungi identified, few had significant impact on nuts contamination under each variable situation. For instance, whenever Alternaria, Botrytis, Cladosporium and Fusarium were widely distributed, Penicillium and Aspergillus were less significant, and vice-versa. And even in those samples where Aspergillus became the major contaminant, less than five sections (SDI) from the entire Aspergillus population (maximum Richness of 8) were of significance for the overall mycobiota.

The low representativeness of Aspergillus in chestnut samples when compared to total fungal contamination reveals that probably this matrix and the environmental conditions in which chestnuts are produced are not suitable for Aspergillus establishment in the presence of other fungi. Chestnuts have high starch and moisture contents (Wareing et al. 2000; Barreira et al. 2010), which give them the potential to support the growth of a large spectrum of fungi. Field fungi such as Alternaria, Cladosporium and Fusarium have ecophysiological conditions clearly different from those of Aspergillus and Penicillium. The first group of fungi is adapted to colder and more humid conditions and the latter have a more xerophilic and thermophilic 
Table 3 Association between fungi responsible for superficial contamination in chestnuts and almonds, as determined by Qui-square test and Phi coefficient, based on differences between observed and predicted co-infection frequencies

\begin{tabular}{|c|c|c|c|c|}
\hline Fungi & Observed & Predicted & Phi & $P$ \\
\hline \multicolumn{5}{|l|}{ Almonds } \\
\hline Penicillium spp. \& Aspergillus spp. & 253 & 250.1 & 0.170 & 0.004 \\
\hline Penicillium spp. \& Sec. Aspergillus & 225 & 218.4 & 0.289 & 0.000 \\
\hline Penicillium spp. \& Sec. Flavi & 139 & 133.7 & 0.191 & 0.001 \\
\hline Penicillium spp. \& Sec. Nigri & 137 & 132.8 & 0.153 & 0.009 \\
\hline Penicillium spp. \& Sec. Versicolores & 100 & 99.1 & 0.034 & 0.560 \\
\hline Penicillium spp. \& Sec. Wentii & 117 & 112.6 & 0.163 & 0.005 \\
\hline Sec. Flavi \& Sec. Aspergillus & 130 & 108.8 & 0.355 & 0.000 \\
\hline Sec. Flavi \& Sec. Nigri & 97 & 66.1 & 0.426 & 0.000 \\
\hline Sec. Flavi \& Sec. Versicolores & 56 & 49.4 & 0.096 & 0.103 \\
\hline Sec. Flavi \& Sec. Wentii & 75 & 56.1 & 0.266 & 0.000 \\
\hline Sec. Nigri \& Sec. Aspergillus & 122 & 108 & 0.234 & 0.000 \\
\hline Sec. Nigri \& Sec. Versicolores & 44 & 49.0 & -0.072 & 0.218 \\
\hline Sec. Nigri \& Sec. Wentii & 62 & 55.7 & 0.089 & 0.130 \\
\hline Sec. Versicolores \& Sec. Aspergillus & 95 & 80.6 & 0.251 & 0.000 \\
\hline Sec. Versicolores \& Sec. Wentii & 54 & 41.6 & 0.183 & 0.002 \\
\hline Sec. Wentii \& Sec. Aspergillus & 110 & 91.6 & 0.314 & 0.000 \\
\hline
\end{tabular}

nature (Rosso and Robinson 2001; Filtenborg et al. 2004; Magan 2006). Under the environment of chestnut production and the matrix characteristics, field fungi are notably more competitive in the presence of other fungi.

Few studies have been devoted to determining fungal contamination of chestnuts, and none has analysed chestnuts originating from the major producing countries (see Rodrigues et al. 2012b for a comprehensive review). These studies confirm that Aspergillus spp. are significantly more relevant in surveys from hot and dry places than from cold and wet places. The environmental conditions of our samples as well as our results compare mostly with the latter.

Sieber et al. (2007) analysed nuts collected from the ground 2 and 7 days after falling and nuts collected from nets fixed below the tree canopy; they did not register significant differences in fungal contamination between harvest methods. In our study, three harvest methods were tested: from the tree, from the ground at the beginning of harvest, and from the ground 3 weeks after the beginning of harvest. Results showed that harvest method had no significant influence on superficial contamination, but internal total contamination varied significantly. Chestnuts collected after up to 3 weeks on the ground were significantly more contaminated internally with overall fungi than samples collected from the tree or from the ground at the beginning of the harvest period. This may have resulted from the fact that the contact of nuts with the wet soil leads to an increased contact with fungal propagules and, under high humidity, fungi can more easily grow and reach the kernel. On the other hand, the fact that aspergilli were found less frequently in nuts collected from the ground reflects the lower competitiveness of these fungi under high humidity, when in the presence of other fungi.

In field-collected almonds, Aspergillus species were found to be important superficial contaminants of almonds, alongside with Cladosporium. Similar results had been reported for field-collected Californian almonds (Phillips et al. 1979; Purcell et al. 1980; Teviotdale and Hendricks 1994; Bayman et al. 2002). In these studies, Cladosporium and/or Alternaria were also considered major contaminants, and Mucor spp. or Rhizopus spp. were also reported. In our case, these two genera were also found but not at significant frequencies. That might be due to different culture conditions, namely the salt concentration on the culture medium being higher in our study (10\% against 6 to $7.5 \%$ in the other studies). Phillips et al. (1979), Purcell et al. (1980) and Bayman et al. (2002) had also referred to Aspergillus spp. at 
Table 4 Association between fungi responsible for internal contamination in chestnuts and almonds, as determined by Qui-square test and Phi coefficient, based on differences between observed and predicted co-infection frequencies

\begin{tabular}{|c|c|c|c|c|}
\hline Fungi & Observed & Predicted & Phi & $P$ \\
\hline \multicolumn{5}{|l|}{ Almonds } \\
\hline Penicillium spp. \& Aspergillus spp. & 69 & 31.8 & 0.746 & 0.000 \\
\hline Penicillium spp. \& Sec. Aspergillus & 64 & 29.3 & 0.710 & 0.000 \\
\hline Penicillium spp. \& Sec. Flavi & 12 & 5.0 & 0.290 & 0.000 \\
\hline Penicillium spp. \& Sec. Nigri & 4 & 1.7 & 0.164 & 0.017 \\
\hline Penicillium spp. \& Sec. Versicolores & 12 & 5.0 & 0.201 & 0.004 \\
\hline Penicillium spp. \& Sec. Wentii & 6 & 2.5 & 0.202 & 0.003 \\
\hline Sec. Flavi \& Sec. Aspergillus & 11 & 4.0 & 0.304 & 0.000 \\
\hline Sec. Flavi \& Sec. Nigri & 1 & 0.2 & 0.116 & 0.093 \\
\hline Sec. Flavi \& Sec. Versicolores & 3 & 0.5 & 0.252 & 0.000 \\
\hline Sec. Flavi \& Sec. Wentii & 2 & 0.3 & 0.204 & 0.003 \\
\hline Sec. Nigri \& Sec. Aspergillus & 4 & 1.3 & 0.197 & 0.004 \\
\hline Sec. Nigri \& Sec. Versicolores & 0 & 0.2 & -0.029 & 0.669 \\
\hline Sec. Nigri \& Sec. Wentii & 0 & 0.1 & -0.024 & 0.729 \\
\hline Sec. Versicolores \& Sec. Aspergillus & 7 & 3.0 & 0.200 & 0.004 \\
\hline Sec. Versicolores \& Sec. Wentii & 1 & 0.3 & 0.105 & 0.129 \\
\hline Sec. Wentii \& Sec. Aspergillus & 4 & 2.0 & 0.121 & 0.079 \\
\hline
\end{tabular}

significant frequencies, and reported section Nigri as the major Aspergillus contaminant, whereas other sections like Circumdati, Flavi, Fumigati and Nidulantes were only rarely isolated. A negligible internal contamination at this stage of almond production had also been reported by these authors.

Contrary to our data, Phillips et al. (1979) and Purcell et al. (1980) mentioned the detection of section Aspergillus at much lower frequencies than section Nigri. Teviotdale and Hendricks (1994) and Bayman et al. (2002) do not even refer to any Eurotium species (or any of their anamorphic counterparts) in their surveys. This was, to some extent, expected in the case of Teviotdale and Hendricks (1994), where culture medium used for fungal isolation (Potato Dextrose Agar) was not adequate for xerophilic fungi, but not in the others, where mycobiota was analysed in a culture medium similar to ours $(6 \%$ or $7.5 \%$ salt agar). It is possible that the higher salt concentration in our culture medium (10\%) gave selective advantage to Eurotium species. The difference might also have resulted from the different environmental conditions and the almonds' $\mathrm{a}_{\mathrm{W}}$ during maturation and harvest. Our results are supported by the study of King et al. (1983), which analysed field-collected almonds with different water activity and observed that $A$. glaucus (anamorph for E. herbariorum) was the major contaminant of almonds with low $\mathrm{a}_{\mathrm{W}}(0.75-0.80)$, whereas $A$. niger was the most frequent species isolated from almonds with $\mathrm{a}_{\mathrm{W}}>0.9$.

Aspergillus have become significant contaminants in storage-collected almonds. This seems to be a reflex of the effect of extreme environmental conditions on fungal distribution. At the end of this period, almonds registered $\mathrm{a}_{\mathrm{W}}$ of approximately 0.63 , which is too low for the majority of species to grow or even survive. Under these conditions, the most xerophilic sections Aspergillus, Versicolores and Wentii (Filtenborg et al. 2004; Hoekstra et al. 2004) were responsible for almost all the contamination. Without the competitiveness of other fungi, a few propagules of these sections were able to transpose the shell barrier and contaminate the kernel.

Similar results in terms of mycobiota evolution throughout storage periods had been observed by others. The mycobiota of sorghum grains (da Silva et al. 2000) and peanuts (Nakai et al. 2008) from Brazil, and kolanuts from Nigeria (Adebajo and Popoola 2003) was analysed throughout storage periods of up to 12 months and, in all cases, progressive increase of Aspergillus and Penicillium incidences was detected along with the expected decrease of other genera like Cladosporium and Fusarium. 
In our survey, sections Aspergillus, Versicolores and Wentii became strong contaminants in storage almonds, and accounted for the majority of fungal contamination. Section Flavi also became significant, whereas other sections like Circumdati, Flavipedes and Nigri were only rarely isolated. It is noteworthy that section Nigri, which was an important Aspergillus contaminant in the field, almost disappeared during the storage period. This was not expected, since almost all reports on mycobiota of almonds and other similar substrates during storage periods refer to this section as one of the major contaminants under storage conditions, alongside with section Flavi, with evident increases being registered throughout the storage period (e.g. Purcell et al. 1980; Adebajo and Popoola 2003; Kaaya and Kyamuhangire 2006). But a few studies (da Silva et al. 2000; Nakai et al. 2008) have reported results more close to ours, where section Nigri was isolated only rarely from long-term storage samples. Studies on unprocessed almonds marketed in California, USA (Joffe 1969; King and Schade 1986; Bayman et al. 2002), Spain (Jimenez et al. 1991) and Saudi Arabia (Abdel-Gawad and Zohri 1993) have also identified remarkably high contamination with both sections Flavi and Nigri.

Numerous studies have been devoted to fungal surveys on nuts, but few studies have tested for associations among fungi on individual seeds or crop parts. Bayman et al. (2002) and Doster et al. (1996) reported a highly significant association between $A$. flavus and A. niger for Californian nuts (pistachios, almonds, walnuts and Brazil nuts) and for Californian figs, respectively. Also, and even though the association is not clearly determined, the majority of studies on Aspergillus surveys on nuts refer to similar incidence rates of both sections Flavi and Nigri (King and Schade 1986; Abdel-Gawad and Zohri 1993; Freire et al. 2000; Singh and Shukla 2008), which suggests that they are, in fact, associated to some degree. This association is probably due to the fact that sections Flavi and Nigri share common habitats and ecophysiological characteristics (Rosso and Robinson 2001; Magan 2006; Klich 2007), so conditions that favour one of these fungi probably favour the other. Bayman et al. (2002) suggest that infection by one Aspergillus species makes a fruit more susceptible to other aspergilli. Results obtained by Phillips et al. (1979) did not reflect the same type of correlation, as they reported that section Flavi was negatively correlated with sections Nigri and Aspergillus.

Bayman et al. (2002) and Doster et al. (1996) reported a negative association between Aspergillus spp. and Penicillium spp. Bayman et al. (2002) postulated that there is probably some degree of antagonism or competitive exclusion between Aspergillus spp. and Penicillium spp. Our results do not support this theory. Also, a study on the antagonistic effect of various fungi against Aspergillus spp., especially section Flavi, detected that growth of aspergilli in the presence of penicilli was not significantly deterred (Phillips et al. 1979). Joffe (1969) observed that fungi with higher $\mathrm{a}_{\mathrm{W}}$ requirements had stronger antagonistic effect over $A$. flavus than those fungi usually associated with dry foods. It is not completely understood if competition is due to antagonistic effects or to the physical and chemical environment. Whatever the case might be, our results corroborate that perception, since samples with high incidences of field fungi were less contaminated with Aspergillus spp. and vice-versa.

\section{Conclusions}

Chestnuts and almonds are different in their intrinsic biological, chemical and physical characteristics and they are produced under different environmental conditions. The conjugation of these factors resulted in different types of fungi identified as dominant contaminants. Chestnuts were more strongly associated with the so-called field fungi like Alternaria, Cladosporium and Fusarium, and almonds were more associated with the storage fungi Aspergillus and Penicillium. Fungal contamination of both matrices was found to be predominantly superficial, as few fungi were able to effectively transpose the shell and infect the kernel.

In almonds, fungi evolved from field to the end of storage. Potentially toxigenic Aspergillus belonging to sections Flavi and Nigri became generally more significant and widespread throughout storage, and were determined to be moderately associated.

Acknowledgments P. Rodrigues was supported by grants SFRH/BD/28332/2006 from Fundação para a Ciência e a Tecnologia (FCT), and SFRH/PROTEC/49555/2009 from FCT and Polytechnic Institute of Bragança, Portugal. 


\section{References}

Abdel-Gawad, K. M., \& Zohri, A. A. (1993). Fungal flora and mycotoxins of six kinds of nut seeds for human consumption in Saudi Arabia. Mycopathologia, 124, 55-64.

Adebajo, L. O., \& Popoola, O. J. (2003). Mycoflora and mycotoxins in kolanuts during storage. African Journal of Biotechnology, 2, 365-368.

Barreira, J. C., Pereira, J. A., Oliveira, M. B. P. P., \& Ferreira, I. C. F. R. (2010). Sugars profiles of different chestnut (Castanea sativa Mill.) and almond (Prunus dulcis) cultivars by HPLCRI. Plant Foods for Human Nutrition, 65, 38-43.

Bayman, P., Baker, J. L., \& Mahoney, N. E. (2002). Aspergillus on tree nuts: incidence and associations. Mycopathologia, $155,161-169$.

da Silva, J. B., Pozzi, C. R., Mallozzi, M. A. B., Ortega, E. M., \& Corrêa, B. (2000). Mycoflora and occurrence of aflatoxin B1 and Fumonisin B1 during storage of Brazilian sorghum. Journal of Agricultural and Food Chemistry, 48, 4352-4356.

Doster, M. A., Michailides, T. J., \& Morgan, D. P. (1996). Aspergillus species and mycotoxins in figs from California orchards. Plant Disease, 80, 484-489.

Filtenborg, O., Frisvad, J. C., \& Samson, R. A. (2004). Specific association of fungi to foods and influence of physical environmental factors. In R. A. Samson, E. S. Hoekstra, \& J. C. Frisvad (Eds.), Introduction to food-and airborne fungi (7th ed., pp. 306-320). Wageningen: CBS.

Freire, F. C. O., Kozakiewicz, Z., \& Paterson, R. R. M. (2000). Mycoflora and mycotoxins in Brazilian black pepper, white pepper and Brazil nuts. Mycopathologia, 149, 13-19.

Gams, W., Christensen, M., Onions, A. H., Pitt, J. I., \& Samson, R. A. (1985). Infrageneric taxa of Aspergillus. In R. A. Samson \& J. I. Pitt (Eds.), Advances in Penicillium and Aspergillus systematic (pp. 55-62). New York: Plenum Press.

Hoekstra, E. S., Samson, R. A., \& Summerbell, R. C. (2004). Methods for the detection and isolation of fungi in the indoor environments. In R. A. Samson, E. S. Hoekstra, \& J. C. Frisvad (Eds.), Introduction to food-and airborne fungi (7th ed., pp. 298-305). Wageningen: CBS.

Jimenez, M., Mateo, R., Querol, A., Huerta, T., \& Hernandez, E. (1991). Mycotoxins and mycotoxigenic moulds in nuts and sunflower seeds for human consumption. Mycopathologia, $115,121-127$.

Joffe, A. Z. (1969). Relationship between Aspergillus flavus, A. niger and some other fungi in the mycoflora of groundnut kernels. Plant and Soil, 31, 57-64.

Kaaya, A. N., \& Kyamuhangire, W. (2006). The effect of storage time and agroecological zone on mould incidence and aflatoxin contamination of maize from traders in Uganda. International Journal of Food Microbiology, 110, 217-223.

King, A. D., \& Schade, J. E. (1986). Influence of almond harvest, processing and storage on fungal population and flora. Journal of Food Science, 51, 202-205.

King, A. D., Halbrook, W. U., Fuller, G., \& Whitehand, L. C. (1983). Almond nutmeat moisture and water activity and its influence on fungal flora and seed composition. Journal of Food Science, 48, 615-617.

Klich, M. A. (2002). Identification of common Aspergillus species. Wageningen: CBS.

Klich, M. A. (2007). Environmental and developmental factors influencing aflatoxin production by Aspergillus flavus and Aspergillus parasiticus. Mycoscience, 48, 71-80.

Magan, N. (2006). Mycotoxin contamination of food in Europe: early detection and prevention strategies. Mycopathologia, $162,245-253$.

Maroco, J. (2003). Análise Estatística - Com utilização do SPSS (2nd ed.). Lisbon: Edições Sílabo, Lda.

Nakai, V. K., Rocha, L. O., Gonçalez, E., Fonseca, H., Ortega, E. M. M., \& Corrêa, B. (2008). Distribution of fungi and aflatoxins in a stored peanut variety. Food Chemistry, 106, 285-290.

Phillips, D. J., Mackey, B., Ellis, W. R., \& Hansen, T. N. (1979). Occurrence and interaction of Aspergillus flavus with other fungi on almonds. Phytopathology, 69, 829-831.

Purcell, S. L., Phillips, D. J., \& Mackey, B. E. (1980). Distribution of Aspergillus flavus and other fungi in several almondgrowing areas of California. Phytopathology, 70, 926-929.

Rodrigues, P., Venâncio, A., \& Lima, N. (2012a). Aflatoxigenic fungi and aflatoxins in Portuguese almonds. The Scientific World Journal. doi:10.1100/2012/471926.

Rodrigues, P., Venâncio, A., \& Lima, N. (2012b). Mycobiota and mycotoxins of almonds and chestnuts with special reference to aflatoxins. Food Research International, 48, 76-90.

Rosso, L., \& Robinson, T. P. (2001). A cardinal model to describe the effect of water activity on the growth of moulds. International Journal of Food Microbiology, 6, 265-273.

Samson, R. A., Hoekstra, E. S., Lund, O., Filtenborg, O., \& Frisvad, J. C. (2004). Methods for the detection, isolation and characterisation of food-borne fungi. In R. A. Samson, E. S. Hoekstra, \& J. C. Frisvad (Eds.), Introduction to foodand airborne fungi (7th ed., pp. 283-305). Wageningen: CBS.

Sieber, T. N., Jermini, M., \& Conedera, M. (2007). Effects of the harvest method on the infestation of chestnuts (Castanea sativa) by insects and moulds. Journal of Phytopathology, 155, 497-504.

Singh, P. K., \& Shukla, A. N. (2008). Survey of mycoflora counts, aflatoxin production and induced biochemical changes in walnut kernels. Journal of Stored Products Research, 44, 169-172.

Teviotdale, B. L., \& Hendricks, L. (1994). Survey of mycoflora inhabiting almond fruit and leaves in conventionally and organically farmed orchards. Acta Horticulturae, 373, 177-183.

Wareing, P. W., Nicolaides, L., \& Twiddy, D. R. (2000). Nuts and nut products. In B. M. Lund, T. C. Baird-Parker, \& G. W. Gould (Eds.), The microbiological safety and quality of food (pp. 919-940). Maryland: Aspen Publication.

Zak, J. C., \& Willig, M. R. (2004). Fungal biodiversity patterns. In G. M. Mueller, G. F. Bills, \& M. S. Foster (Eds.), Biodiversity of fungi: Inventory and monitoring methods (pp. 59-75). Waltham: Elsevier Academic Press. 